\title{
Modern information systems as a marketing tool in the development of a tourism business
}

\author{
Oxana Katerinich $^{1}$, Tatiana Degtyaryova ${ }^{2}$, and Irina Usova ${ }^{1 *}$ \\ ${ }^{1}$ Institute of Technologies (branch) of DSTU in Volgodonsk, Mira avenue, 16, Volgodonsk, Rostov \\ region, 347386, Russia \\ ${ }^{2}$ Institute of Service Sector and Entrepreneurship (branch) of DSTU, Shevchenko street 147, Shakhty, \\ Rostov region, 346500, Russia
}

\begin{abstract}
This scientific and theoretical article is devoted to the consideration of the problem of the tourism which can be called one of the most innovative and automated industries in the modern economy. To simplify complex processes in the tourism industry, there are many innovative and high-tech tools that are constantly updated focusing on the needs of the audience and changing in accordance with them. The novelty of the article is due to its applied orientation.
\end{abstract}

\section{Introduction}

In general tourism software can be categorized as follows: systemic, applied: standard / special, booking systems - global, local, corporate, as well as hotel broker systems and online booking systems, tourism automation systems or client services [1].

The list of information technology commonly used in the tourism industry, including a list of software and information reference systems, can be summarized as follows:

- Windows operating system and Microsoft Office applications (systems such as Mac and Linux can also be used in work, but they are owned by a much smaller number of users);

- Reference and legal system «ConsultantPlus»;

- Information and legal system «Legislation of Russia»;

- Federal State Information System «National Electronic Library»;

- $\quad$ Register of objects of the tourism industry [2].

As a sphere of economic and entrepreneurial activity, information and intercultural communication, tourism is an area where the specificity, scale and interconnectedness of all modern processes and trends of world globalization are very clearly manifested.

Communication, information, production, marketing, educational tourism space is now fully covered by globalization processes that affect all spheres of modern life, including tourism policy, economy, technology, education.

Thanks to such globalization, the rapid development of information technology, as well as the high speed of information transfer, today the most important principle of not only

\footnotetext{
*Corresponding author: irina_usova@mail.ru
} 
tourism, but any business, is the speed of customer service, presence on the Internet and the use of the most modern technologies [3].

\section{Innovative technological systems using in tourism industry}

To ensure the activities of the objects of the tourism industry today, it is vital to own and use various innovative technological systems that make it possible to facilitate and simplify the daily tasks facing the business.

Many of the processes that used to be performed manually and took a certain amount of time, today it is possible to automate using various software and thereby significantly speed up.

For example, the accounting of a modern company is impossible without the use of programs such as $1 \mathrm{C}$ Accounting or ConsultantPlus, which allow you to quickly and easily organize all financial data, correctly and on time to draw up the necessary documentation.

To find clients and advertise a tourist product or company in today's realities, you need to be able to use the Internet and various social media. If a travel company today is not present on such social networks as facebook.com, instagram.com, airbnb.com, booking.com, then it is unlikely that a potential client will be able to find it. It is much more convenient for the consumer to find out all the information in advance by examining the official product pages [4]. Also, these networks have a unique property to initially set up a potential client in a friendly way - he understands all offers and prices, the mechanisms of communication with the company are obvious, it is interesting to view the presented photographs of tourist products and destinations, to find out useful information through blogs and stories. Receiving all the necessary information from an active source, the user is pre-configured in a friendly way, unlike standard sites, which are an excellent business card, but as a rule, they do not provide all the latest information in an understandable format and the required volume.

Google tools stand out, as well as a huge variety of different applications for mobile devices that offer users a very wide range of additional options.

It is worth noting the emergence of special new professions associated with ensuring the correct management of social networks: copywriters who create attractive and interesting content, and most importantly, the content needed by a potential client - live and understandable texts, selection of attractive pictures and videos; SMM managers who create a general concept for social media, prescribe content plans for copywriters; targeting experts who allow you to set up advertising correctly, use the advertising budget wisely, attracting a specific audience [5]. Today, if a company is not on social networks, then it simply does not exist on the market. It is very unlikely that the client will independently enter the office from the street - the habit has already developed to get acquainted with the product virtually, read reviews, view photos and the cost of services, as well as booking and paying for services in advance online.

The active development of Internet technologies has led to the introduction of such an innovation as online tourism. The latest world events related to the epidemiological situation and the suspension of tourist traffic around the world, although it brought down the tourist market as a whole, at the same time pushed the development of such new directions as online excursions, various video projects, video blogs, online exhibitions[6]. This is especially true of art tourism and the area of cultural heritage - monuments of architecture and culture. If sports or beach tourism is difficult to present online, then thanks to the development of technology, today you can easily visit the Louvre or the Sistine Chapel without leaving your home [7]. The updated sites of many of the most important cultural and historical destinations today contain comprehensive information, including 
online lectures, 3D video excursions, detailed encyclopedic references and other materials that allow the virtual traveler to get the most out of the cultural and historical heritage.

Of course, even the highest quality $3 \mathrm{D}$ excursion cannot compare with a visit to a real cultural, historical or art object, it will not replace a trip to a theater or museum, and will not allow you to truly enjoy the majesty of cathedral architecture. However, the development of online platforms has a number of undeniable positive qualities. First of all, this concerns the discovery of unique additional opportunities for those people who, due to their own limited physical and other capabilities, previously could not get acquainted with the world monuments of culture and history [8]. Thanks to the powerful development of online platforms, this vulnerable group of the population also has a chance to join the most important monuments of the world cultural and historical heritage, as well as to get acquainted with new forms of art. Another plus in the development of online platforms is an excellent opportunity for tourists to get to know objects and destinations well in advance, and plan their future trips more carefully.

In modern conditions of economic development, characterized by globalization, continuity, stability and acceleration, it is increasingly important to increase the competitiveness of organizations in the real sector of the economy. For the full development of any tourist organization in the long term, the organization needs the ability to anticipate changes in the market in time, quickly adapt its organizational structure and the content of the portfolio of orders for travel services to them [9].

Therefore for the stable and full-fledged development of any enterprise in the field of tourism, permanent monitoring of the environment, constant analysis of the audience, alternative technologies, social changes and legislation that represent either favorable opportunities or threats to business is vital.

One of the most important monitoring methods necessary for the correct functioning of a tourist organization is the method of sociological research: sociological polls of focus groups, interviews with experts, questioning of local residents and vacationers. The experts can be representatives of professional associations, tour operator companies, government bodies of the federal and regional levels.

Another no less important method is the analysis of the flow of public information: various media, the Internet. This method makes it possible to assess the attractiveness of a region among potential consumers in the information space on the basis of semantic constructions describing tourist and recreational activities in relation to a certain territory [10]. The contribution of tourism to the brand of the region, the activity of the administration, business and the population in the development of the tourism industry, the positive and negative aspects of the state of the tourist infrastructure, the readiness of the local population to be involved in the process of receiving and serving tourists can also be assessed.

And one of the most important tools is the method of comparing the final results. As a result of mathematical processing of statistical data and opinion polls, one can consider, for example, a ranked list of OTEs. By comparing the two lists, it is possible to identify a correlation between them, on the basis of which to make decisions about the profitability of certain measures for the development of tourist and recreational activities in a given region for this project.

Consider one of the newest tools - automated polls, for example, Google polls [11].

Such forms are very convenient for compiling various types of surveys and can be useful both for conducting marketing research of the market and for other purposes, for example, for questioning potential service personnel, collecting data to find the necessary professionals, to receive feedback from tourists who have already visited the facility. or to investigate the potential of a product $[12,13]$. This very simple and convenient modern tool for collecting information and analyzing the data obtained is available to any user for free. 
Such questionnaires allow you to conveniently collect the necessary preliminary data, including from potential customers, to understand the attitude to a particular issue, and also allow you to systematize and analyze the data obtained automatically. You no longer need to calculate the percentage of those who answered one way or another to each question, the system calculates and sorts everything automatically.

Consider the operation of this innovative technological system. To create surveys using Google Forms, you first need to create a Google account, open GoogleDrive and create a new GoogleForms document (Fig. 1).

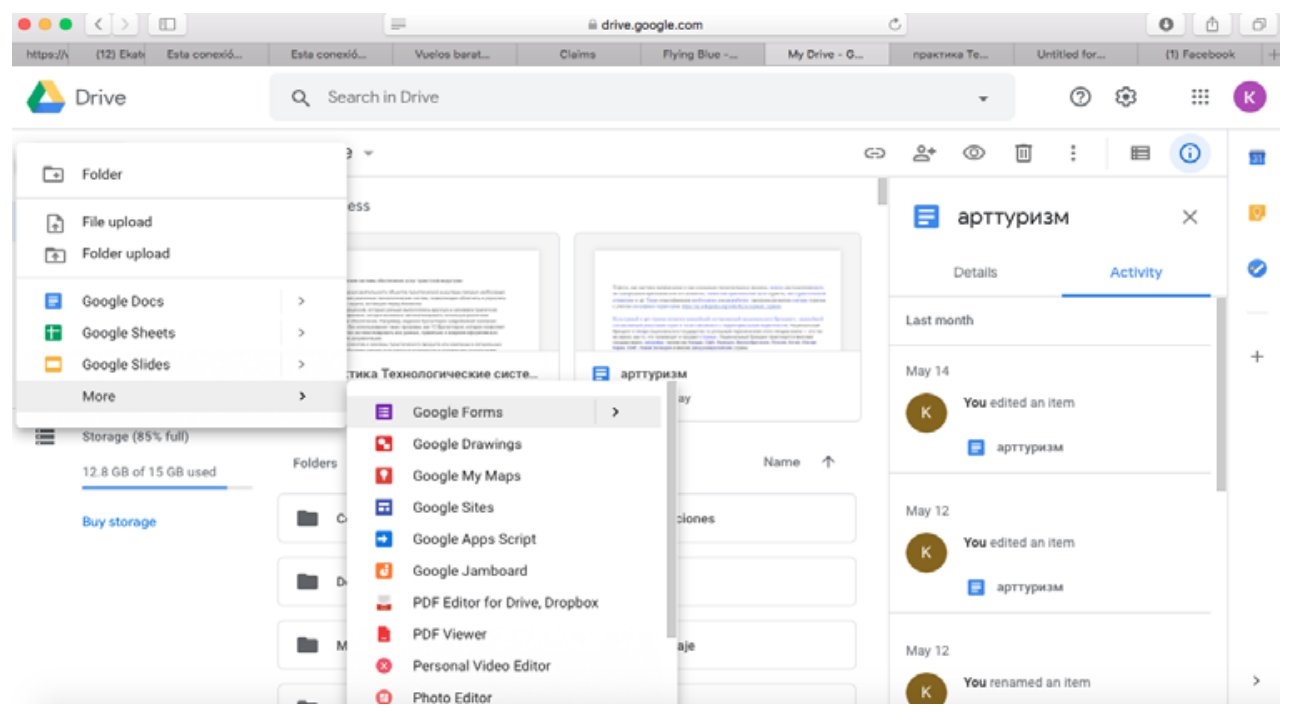

Fig. 1. Create a GoogleForms survey.

Using the settings, you can give the survey the desired look by choosing the background color and fonts, uploading images, choosing the desired form of answers: short answer, description, choosing one answer from the listed ones, choosing several options, marking the question as mandatory, and so on (Fig. 2).

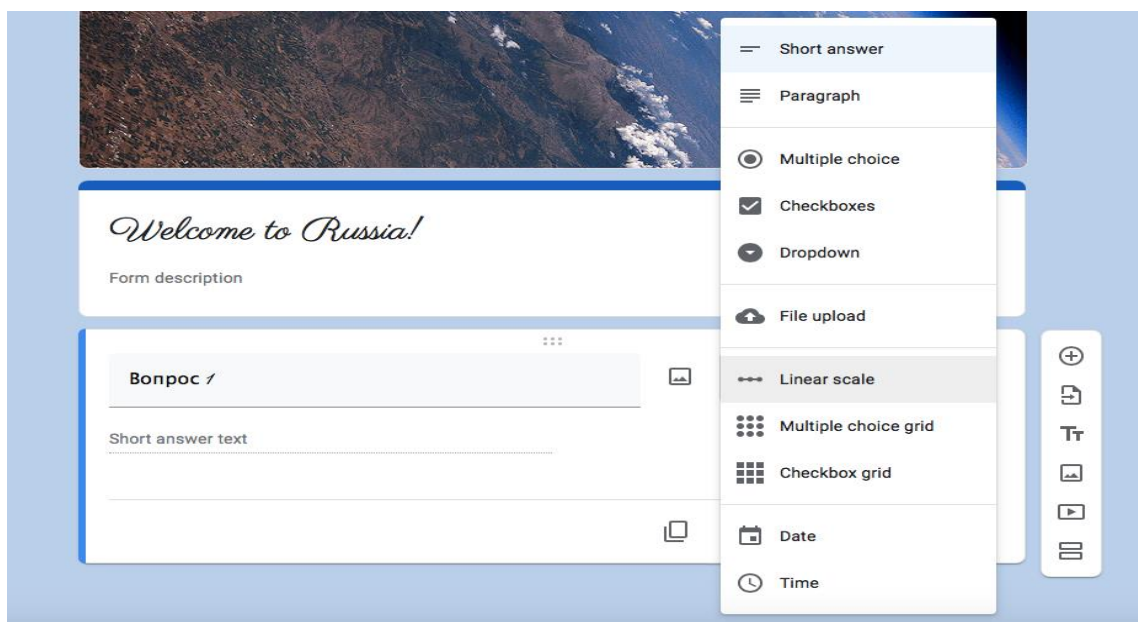

Fig. 2. Editing GoogleForms Surveys. 
When all the questions are ready, the survey can be shared using the link - by sending it by e-mail, by sending a personal message to any messenger or by reposting on social networks [14].

All answers will be automatically saved and analyzed by the system, resulting in convenient diagrams, graphs, reports. Also, the answer of each participant can be viewed separately or downloaded as a separate file [15].

\section{Conclusion}

Thanks to the use of modern innovative technologies and tools, such as automated online surveys, it is possible to easily understand the requests, problems and fears of potential customers, quickly structure responses, and understand which products and services are relevant at the moment, which areas of business in tourism need to be given special attention based on what characteristics to correct the image of the destination and the country as a whole.

Monitoring systems such as Google Forms polls allow you to understand a potential consumer much better, get feedback from him, based on which you can provide the market in advance with all the products and services necessary for a comfortable stay of a tourist, improve the quality of services provided, and develop new tools and products that are able to bring new income, jobs and investments to the regions of Russia.

\section{References}

1. O. Katerinich, T. Degtyaryova, I. Stolyar, I. Usova, 3S Web of Conferences 210, 12003 (2020)

2. D.A. Kozlov, Bulletin of the Russian University of Economics. G.V. Plekhanov, 12(66), 49-56 (2017)

3. F. M. Y. Roxas, J. P. R. Rivera, E. L. M. Gutierrez, Journal of Hospitality and Tourism Management, 45, 387-398 (2020).

4. V.P. Kazachinsky, G.V. Stepanovskaya, Z.M. Khasheva, O.L. Shulgaty, International Journal of Economic Perspectives, 3, 1686-1696 (2018)

5. I.N. Gavrilchak, E.E. Sharafanova, Y.G. Terentyeva, Russian Journal of Entrepreneurship, 12 (84), 97-100 (2011)

6. L. Zhenpeng, Y. Wang, E. Marnburg, Journal of Hospitality Marketing and Management, 22(6), 656-677 (2013)

7. N. Gunasekaran, S. Peter, J. George, S. V. Anandkumar, International Journal of Management, 3(3), P. 8-16 (2012)

8. S. Foxley, Journal of Property Investment \& Finance, 19(1), 79-89 (2011)

9. M. G. Repina, Bulletin of the Russian International Academy of Tourism, 3, 11-14 (2014)

10. R.G. Fedorov, E.A. Kosheleva, Russian Journal of Entrepreneurship, 6 (228), 127-132 (2018)

11. A.L. Beloborodova, O.V. Martynova, International Journal of Economic Perspectives, 3, 1870-1876 (2017)

12. A.V. Bodrov, Russian Journal of Entrepreneurship, 7, 155-159 (2018)

13. I.N. Gavrilchak, E.E. Sharafanova, Yu.G. Terentyeva, Russian Journal of Entrepreneurship, 12 (84), 97-100 (2011) 
14. E.S. Akopova, N.V. Przhedetskaya, K.V. Borzenko, International Journal of Economic Perspectives, 4, 416-421 (2016)

15. A.A. Larionova, N.A. Zaitseva, T.V. Degtyaryova, O.S. Mysova, N.G. Nagai, N.T. Oboimova, N.V. Shcherbakova, Modern Journal of Language Teaching Methods, 10, 630-641 (2018) 